(SIDS). The aim of this study was to determine if evidence suggests that commercially available sleep movement monitors should be routinely recommended by healthcare professionals. Methods A systematic literature review was undertaken to investigate the evidence for the efficacy of infant sleep monitors. The articles retrieved were then screened in accordance with the Preferred Reporting Items for Systematic Reviews and Meta-Analyses (PRISMA) guidelines.

Results Literature search yielded five relevant articles, a majority $(80 \%)$ relating to SIDS. Two studies showed the monitor was effective in accurately detecting cessation of breathing but could not comment on their efficacy with regards to SIDS prevention. A study of 53 infants using the Babysense monitor after an Apparent Life-Threatening Event (ALTE) found the monitor accurately detected apnoea and bradycardia when compared to the cardiorespiratory monitor 'Intellivue MP20 Junior' by Phillips. Two qualitative studies reported that such devices were appealing to mothers.

Discussion The medical effectiveness and reliability of these movement monitors is still a matter of controversy. Commercial monitors may be comparable to clinical cardiorespiratory monitors in terms of detection of apnoea and bradycardia. However, no article could conclude that sleep movement monitors are an effective method of SIDS prevention. Instead, healthcare professionals should emphasise interventions proven to reduce the risk of SIDS such as positioning infants on their back to sleep, or smoking cessation. Further limitations of the devices included a high rate of false alarms.

Conclusion The systematic review revealed that there is no evidence that commercially available sleep movement monitors can prevent SIDS. Therefore, sleep movement monitors should not be routinely recommended by paediatricians. However, some of the studies have shown the potential for other uses for these monitors. There is some evidence to suggest that they may be of use for monitoring specific cohorts of infants, including those who have had a previous ALTE, or have cardiorespiratory risk factors. Further research into these areas is required.

\section{GP173 USAGE OF PROBIOTICS IN THE TREATMENT OF GASTROENTERITIS IN THE PAEDIATRIC POPULATION - A SYSTEMATIC REVIEW}

Allison Bell, Laken Boochoon, Craig Duffy, Jia Jun Foo, Sarah Koscic, Nnedimma Ozoani, Abidur Rahman, Darolyn Tan, Eleanor Molloy*. Department of Paediatrics, Tallaght University Hospital, Trinity College Dublin, Dublin, Ireland

\subsection{6/archdischild-2019-epa.234}

Background Acute gastroenteritis is defined as a sudden onset of diarrhoea which in itself is the passage of 3 or more loose stools in a 24 hour period, or the passage of one or more bloody stools in the presence or absence of abdominal pain, fever, nausea and vomiting. Acute gastroenteritis is self-limiting lasting no longer than 2 weeks, however, it is a major cause of morbidity and mortality worldwide. Current primary treatment modalities include restoration of the acid-base balance, correction of electrolyte disturbances, and oral rehydration therapies. The aforementioned therapeutic regimens help to decrease morbidity and mortality, however, it seldom has an impact on the duration of the infection and its symptoms. Probiotics are believed to help reduce both the duration and severity of symptoms of gastroenteritis, however strain specific efficacy and inter-strain comparison has not yet been established.

Objective The aim of this systematic review was to examine established original research to determine the efficacy of probiotics in treating acute gastroenteritis in the paediatric population. We examined the different strains of probiotics utilized and their associated outcomes in treating gastroenteritis.

Methods An Embase search was carried out with the help of a medical librarian. Two independent reviewers screened title and abstract, followed by full text review using the programme Covidence. All reviewer conflicts were resolved by a third party. Data was extracted from the included articles to determine correlation and effects of probiotics.

Results Of the 581 results obtained from the search, 11 studies were included for data extraction after applying the inclusion and exclusion criteria. Majority of the studies showed probiotics reduced duration of diarrhoea (8 of 11 studies), and a reduced duration of hospitalisation (6 of 11 studies). Notably, 2 papers reported adverse effects, such as fungaemia, in immunocompromised and ICU patients. The different strains of probiotics that were examined in the selected papers include Saccharomyces boulardii, Lactobacillus casei, and Lactobacillus acidophilus.

Conclusion Probiotics reduced the duration diarrhoea symptoms and hospitalization. Usage of probiotics was however accompanied by minimal side effects but not indicated in immunocompromised patients with gastroenteritis. However, further research needs to be conducted to determine the strain specific efficacy and dosage requirements for treatment of gastroenteritis using large scale double blinded randomized control trials.

\section{GP174 ALLERGY-FOCUSED HISTORY QUESTIONNAIRE AND ASSESSMENT OF GENOTYPE OF POLYMORPHIC MARKER RS182549 IN THE MCM6GENE ALLOW TO OPTIMIZE THE DIET FOR CHILDREN WITH INFLAMMATORY BOWEL DISEASES}

${ }^{1}$ Irina Gordeeva, ${ }^{1,2}$ Svetlana Makarova*, Kirill Savostyanov ${ }^{1}$, Leyla Namazova-Baranova ${ }^{2}$ Alexandr Pushkov 1, Dasha Golubova 1, Andrey Surkov 1, Oksana Ereshko 1, Maria Golubova '. 'Federal state autonomous institution National Medical Research Center for Children's Health Ministry of Health Russian Federation, Moscow, Russian Federation; ${ }^{2}$ Pirogov Russian National Research Medical University, Moscow, Russian Federation

\subsection{6/archdischild-2019-epa.235}

Children with inflammatory bowel disease (IBD) often receive a dairy-free diet during remission period without evidence, which adversely affects their nutritional status.

The aim Of the study was to optimize the approaches to prescribing diets for children with IBD

Materials and methods 180 children aged 1 to 17 years with IBD (90 patients with Crohn's disease and 90 - with ulcerative colitis) in clinical remission were included in this study. Testing of Lactase deficiency included Lactose Intolerance quick test (LIQT) and Real-time PCR, using fluorescent TaqMan probes for analyzing the genotype of polymorphic marker $r s 182549$ in the MCM6 gene. Allergy-focused history questionnaire, morphological study of biopsy specimens of the intestine with the counting of eosinophils, dairy products «open food challenge» (with fecal calprotectin assessment) were used for identifying of cow's milk allergy (CMA). 
Results According to the results of LIQT: normolactasia (NL) was observed in 26\%, moderate hypolactasia (GL) - in 35\% and severe GL - in 39\% patients with Crohn's disease. In children with Ulcerative colitis NL was observed in $31,5 \%$, moderate GL - in 32,5\% and severe GL - in 36\%. In the patients with normolactasia we revealed an increase in the number of genotypes $T T$ of the of the polymorphic marker rs182549 (c.13910 C>T). In patients with moderate and severe GL, the genotype CC of this polymorphic marker was significantly more frequent $(\mathrm{P}=0.030 ; \mathrm{OR}=2.72 ; 95 \% \mathrm{CI}$, 1.08-6.87).

Dairy products «open food challenge» was performed in 20 children with IBD and suspected CMA (questionnaire score 54-71). CMA was confirmed in 12 patients by the results of the morphological study of intestinal biopsy specimens with the counting of eosinophils and «open food challenge». According to these findings diet recommendation for patient with IBD were personalized: Cow's milk-free diet for children with CMA, Lactose-free diet - for children with severe lactose intolerance, Low lactose diet was recommended to the patients with moderate hypolactasia, Diet containing Cow's milk - for other patients. Children with GL and genotypes TT had better prognosis for lactosetolerance.

Conclusion Allergy-focused history questionnaire for identifying of CMA, Lactose Intolerance quick test and assessment of genotype of polymorphic marker rs182549 in the MCM6 gene may improve approaches for personalization of diet for patients with IBD.

\section{GP175 MUCOSAL ATROPHY AND CLINICAL CORRELATION IN CHILDREN WITH ULCERATIVE COLITIS}

Lorraine Stallard*, Seamus Hussey. Department of Gastroenterology, Our Lady's Children's Hospital Crumlin, Dublin, Ireland

\subsection{6/archdischild-2019-epa.236}

Background Mucosal healing is now preferred over clinical remission as a target endpoint in the treatment of Ulcerative Colitis (UC) as it has been demonstrated to reduce hospitalisation and corticosteroid use, to reduce the risk of colectomy and to reduce the risk of colorectal cancer. In routine practice, the activity and severity of UC is based on combined clinical and endoscopic assessment in addition to histology. However, it is not clear whether the currently available histologic indices can help predict disease course and treatments. Mucosal atrophy (MA) describes a state of extensive mucosal architectural damage, causing irregularities in the morphology and distribution of the intestinal glands of the mucosa. The aim of this study was to investigate the prevalence of MA in a paediatric cohort of newly diagnosed patients with UC and evaluate the relationship between the presence of MA, disease activity and treatments.

Methods Children $<17$ years old diagnosed with UC and recruited to the DOCHAS study (Determinants and Outcomes in Children and Adolescents with IBD) between January 2012 and December 2016 were included in the study. Colonic biopsies were evaluated for the presence of MA at time of diagnosis in treatment naïve patients with UC. Correlation with clinical indices was performed using the Paediatric Ulcerative Colitis Activity Index (PUCAI). Follow up data including
PUCAI, current treatment and relapse rates were collected at 3 months, 6 months, 12 months and yearly thereafter.

Results 176 patients with UC were included of which 21 (12\%) had MA on biopsy at diagnosis (mean age 11.9 years, $51 \%$ female). Those with MA had higher PUCAI scores at three month $(p=0.04)$, six month $(p=0.01)$ and two year $(\mathrm{p}=0.01)$ follow up. MA patients had significantly higher rates of progression to immunomodulators $(58 \%$ vs $44 \%)$ and biologics $(25 \%$ vs $11 \%)$, were more likely to have a clinical relapse $(p=0.04)$ and had shorter times to first relapse post diagnosis $(p=0.01)$.

Conclusion Children with UC and mucosal atrophy demonstrate a more severe disease course with higher disease activity scores and rates of relapse, which results in increased rates of progression to more intensive therapies. Further research into the mechanisms underlying the more severe disease course of mucosal atrophy patients is warranted.

\section{GP176 MORPHOLOGICAL FEATURES OF CHRONIC GASTRITIS ASSOCIATED WITH EPSTEIN-BARR VIRUS IN CHILDREN}

${ }^{1}$ Valeria Novikova*, ${ }^{1}$ Olga Gurina, ${ }^{1}$ Olga Varlamova, ${ }^{1}$ Aleksey Blinov, JeanClaude Hakizimana ${ }^{1},{ }^{1}$ Yulia Karpeeva, ${ }^{2}$ Andrey Petrovsky. ${ }^{1}$ St. Petersburg State Pediatric Medical University, Saint Petersburg, Russian Federation; ${ }^{2}$ North-Western State Medical University named after I.I. Mechnikov, Saint Petersburg, Russian Federation

\subsection{6/archdischild-2019-epa.237}

Introduction The Epstein-Barr virus has been recently considered as an etiological cause of chronic gastritis.

Objectives To study the relationship between the morphological features of chronic gastritis and Epstein-Barr virus infection.

Methods 50 children (mean age $12.4 \pm 0.3$ years) with morphologically verified chronic gastritis were examined. In All patients, fibrogastroduodenoscopy was carried out and two biopsy samples from the body and antrum parts of the stomach were taken. Morphological assessment of biopsy specimens was done according to a standard visual analogue scale. The EBV was determined by immunohistochemical method. The EBV antibodies anti-EA IgG and anti-EBNA IgM were determined by ELISA using standard sets manufactured by 'Vector Best' company. Statistical processing of research materials was carried out using software and package Statistica for Windows (version 6.0).

Results Anti-EA IgG to EBV, indicating active virus replication, were identified in 36 children (72\%). Antibodies to EBV class IgG $\mathrm{M}$, characteristic of the acute stage of EBV infection, had not been found in any child. EBV was detected in $71.4 \%$ of biopsy specimens from the stomach of the children who had been tested anti-EA IgG positive. The presence of EBV antigens in the gastric epithelium revealed significant correlation with the presence of hemorrhages in the body of the stomach $(\mathrm{r}=0.68 ; \mathrm{p}<0.05)$ and in the antrum $(\mathrm{r}=0.66 ; \mathrm{p}<0.05)$, microthrombosis in the body of the stomach $(\mathrm{r}=0.38 ; \mathrm{p}<0.05)$ and in the antrum $(r=0.42 ; \mathrm{p}<0.05)$, regenerative changes in the cervical epithelium of the gastric mucosa $(r=0.32 ; p<0.05)$, fibrosis in the mucosa of the body of the stomach $(r=0.46$; $\mathrm{p}<0.05$ ), atrophy of the glands of the body of the stomach $(\mathrm{r}=0.65 ; \mathrm{p}<0.05)$, atrophy of antral glands $(\mathrm{r}=0.52 ; \mathrm{p}<0.05)$, pylorization of fundal glands $(\mathrm{r}=0.5 ; \mathrm{p}<0.05)$, hyperplasia of parietal cells $(r=0.62 ; \mathrm{p}<0.05)$, erosion in the body of the stomach $(\mathrm{r}=0.47 ; \mathrm{p}<0.05)$, erosion in the antrum $(\mathrm{r}=0.36$; $\mathrm{p}<0.05)$. 